\title{
Kurzbeiträge
}

Markus Hilpert*

\section{Touristische Wertschöpfung kleiner und mittlerer Wallfahrtsorte}

\author{
Regionalökonomische Bedeutung katholischer Heiligtümer ${ }^{1}$ \\ im ländlichen Raum
}

https://doi.org/10.1515/tw-2020-0010

\section{Einführung}

Wallfahrtsorte sind seit jeher Destinationen für gläubige Menschen, weshalb das Wallfahren vermutlich "die älteste Form des Massentourismus" (Imkamp 2010) ist. Seine wirtschaftliche Relevanz generiert dieser Religionstourismus vor allem durch ergänzende Infrastrukturen, wie Einkehr-, Übernachtungs- oder Versorgungseinrichtungen an den Wallfahrtsorten und entlang der Reiserouten (z.B. Jakobuspilgerweg). Daher wurde schon früh die regionalökonomische Bedeutung von Wallfahrtsstätten erkannt. Mittlerweile hat sich das Wallfahrtswesen aber vielfach verändert. Gerade die sozioökonomischen Transformationen in der zweiten Hälfte des 20. Jahrhunderts verursachten einen tiefgreifenden Wandel (Casanova 2006): Durch die Massenmotorisierung verlor zunächst die fußläufige Erreichbarkeit der Wallfahrtsorte an Bedeutung und die soziale Säkularisierung (z.B. Entkirchlichung) führte schließlich zu einem weiteren Bedeutungsverlust vieler Wallfahrtsorte. In jüngster Vergangenheit lässt sich allerdings wieder ein zunehmendes Interesse an Spiritualität nachweisen (Davie 2008; Woodhead 2016). Der Bestseller „Ich bin dann mal weg“ des TV-Moderators Hape Kerkeling ist ein Ausdruck dieses neuen Trends, wenngleich dieser Prozess keine simple Rückkehr zur früher geübten Praxis des Wallfahrens ist (Sommer 2012; Edles

1 Das kanonische Recht der katholischen Kirche verwendet nicht den Begriff Wallfahrtsort, sondern spricht stattdessen von einem Heiligtum, das ,aus besonderem Frömmigkeitsgrund [von] zahlreichen Gläubigen mit Gutheißung des Ortsordinarius“ aufgesucht wird.

*Corresponding author: PD Dr. Markus Hilpert, Universität Augsburg, Institut für Geographie, Alter Postweg 118, D - 86159 Augsburg, E-Mail: markus.hilpert@geo.uni-augsburg.de 
2015). Die Motive zum Besuch eines Wallfahrtsortes umfassen heute auch Sport (z. B. Wandern), Erholung, Kultur, Kunst, Bildung Architektur, Malerei oder Bildhauerei (Pack 2010; Marine-Roig 2015). Solch profane Aspekte ermöglichen aber auch die Fokussierung nicht- und wenig-gläubiger Zielgruppen (Wiltshier \& Griffiths 2016).

Von den Ausgaben der Besucher von Wallfahrtsorten profitieren mehrere Branchen (v. a. Übernachtungs-, Beherbergungs- und Gastronomiebetriebe sowie Versorgungs- und Serviceeinrichtungen). Daten zur regionalökonomischen Bedeutung liegen bislang aber nur für Wallfahrtsorte mit supranationaler Bedeutung vor (Graave et al. 2017), für kleiner und mittelgroßer Wallfahrtsorte, die nur ein regionales Einzugsgebiet aufweisen, existieren bislang aber kaum belastbaren Zahlen. Am Beispiel von vier Wallfahrtskirchen in Bayerisch-Schwaben wurden deshalb Daten zur Wertschöpfung erhoben und analysiert.

\section{Methodik}

An vier kleinen bzw. mittelgroßen Wallfahrtsorten wurden standardisierte Besucherbefragungen an unterschiedlichen Wochentagen durchgeführt. Im Zentrum der Interviews standen Fragen zum Ausgabeverhalten, zu Besuchsmotiven, zur Aufenthaltsdauer und zu additiven Aktivitäten in der Region. Der kleinste untersuchte Wallfahrtsort ist die St. Jakobus-Kirche in Biberbach (ca. 10.000 Besucher jährlich), wo 25 Besucher in einer Pilot- und 100 in der Hauptstudie befragt wurden. In Wigratzbad (ca. 15.000 Besucher jährlich) wurden 200 Besucher befragt, 50 davon in der Pilotstudie. An der Wallfahrtskirche Maria Brünnlein (ca. 150.000 Besucher jährlich) in Wemding wurden 200 Besucher und in Maria Vesperbild (ca. 450.000 Besucher jährlich) wurden 162 Besucher befragt.

Die, durch die Besucher induzierte Wertschöpfung wurde über ein Verfahren berechnet, das sowohl die Berechnung direkter und indirekter Einkommenseffekte, als auch die Schätzung von Beschäftigungseffekten ermöglicht (Rütter et al. 1996; Metzler 2007). Dabei werden aus den Ausgaben der Besucher und der jährlichen Besucherzahl der annuelle Brutto- sowie (abzüglich der Steuer) der annuelle Nettoumsatz berechnet. Mit einer Wertschöpfungsquote der Gastronomie von 45\% (Maschke 2005) kann folglich die Einkommenswirkung der 1. Umsatzstufe geschätzt kann. Die 2. Umsatzstufe beinhaltet alle indirekten Einkommenswirkungen aus den Vorleistungen (z.B. Zulieferung von Waren, Investitionen für die Substanzerhaltung etc.). Für die Berechnung dieser Einkommenswirkungen wird die Wertschöpfungsquote mit $30 \%$ angenommen. Aus 
der Addition beider Einkommenswirkungen kann letztlich nicht nur der gesamte Einkommenseffekt, sondern auch der Beschäftigungseffekt jedes Wallfahrtsortes geschätzt werden, indem auf Basis der amtlichen Statistik (Primäreinkommen privater Haushalte pro Kopf in der jeweiligen Region) die Einkommensäquivalente rechnerisch ermitteln werden.

\section{Ergebnisse der Fallstudien}

Bei der kleinen Wallfahrtskirche in Biberbach gibt es weder Devotionaliengeschäfte noch Wallfahrtsgaststätten oder Beherbergungsbetriebe, weshalb über zwei Drittel der Besucher (68\%) vor Ort auch kein Geld ausgeben. Dieser hohe Anteil ist auch der Tatsache geschuldet, dass es im Ort lediglich eine Gaststätte gibt, die folglich allein von den jährlichen Gesamtausgaben der 10.000 Besucher der Wallfahrt in Höhe von $29.500 €$ profitiert. Abzüglich von Steuern und Vorleistungen induziert die kleine Wallfahrtsstätte eine lokale Wertschöpfung von rund $14.800 €$ jährlich, die der Gaststätte als Gewinn oder für Löhne und Gehälter zur Verfügung stehen (Hilpert 2018). Daraus ergibt sich mathematisch ein Beschäftigungsäquivalent von etwa einem halben Arbeitsplatz, der durch die Ausgaben der Besucher der Wallfahrtskirche generiert werden könnte, die in der Region vor allem wegen ihrer barocken Architektur und Innenausstattung bekannt ist. Deshalb wird die Kirche an Werktagen auch fast ausschließlich von profanen Touristen besucht, entsprechend ist die Kirchenbesichtigung (69\%) das häufigste Besuchsmotiv.

Wigratzbad bietet den jährlich rund 15.000 Besucher neben der Wallfahrtskirche auch Devotionalienläden, einen Beherbergungs- und mehrere Gastronomiebetriebe sowie ein Seminarhaus. Das breite Wallfahrtsangebot umfasst Gottesdienste, Anbetungen, Prozessionen, Einkehrtage und Exerzitien, weshalb die meisten Besucher religiös motivierten sind. Der Hauptbesuchsgrund ist die Teilnahme an einem Gottesdienst (61\%), profane Motive werden kaum genannt. Mehr als drei Viertel der Besucher (78\%) übernachten in Wigratzbad. Wegen diesem hohen Anteil sind auch die jährlichen Ausgaben mit $431.000 €$ relativ hoch. Von diesen profitieren sowohl die lokale Gastronomie und die Übernachtungsbetriebe, als auch der Devotionalien- und der Einzelhandel (z. B. Lebensmittel), das Verkehrswesen und der Dienstleistungssektor (z. B. Seminare) (Wagner 2017). Abzüglich der Steuern und der Vorleistungen induziert die Gebetsstätte eine Wertschöpfung von rund $187.000 €$, die den Betrieben als Gewinne oder für Löhne und Gehälter zur Verfügung stehen. Daraus ergibt sich mathematisch ein Beschäftigungsäquivalent von fast sieben Arbeitsplätzen. 
Der Wallfahrtsort Maria Brünnlein in Wemding umfasst neben der Wallfahrtsbasilika ein Pilgerheim, einen Gasthof mit Pension, ein Devotionaliengeschäft und das Kerzenhaus. Bekannt ist die Wallfahrtskirche für ihre Innengestaltung im Stil des Rokokos. Entsprechend ist die Kirchenbesichtigung (41\%) der häufigste Besuchsgrund (Laznik 2019). Von den jährlichen Gesamtausgaben der etwa 150.000 Besucher in Höhe von rund 5.539.000€ profitiert in erster Linie die lokale Gastronomie, allerdings fließen auch jeweils ein Fünftel der Ausgaben in die Hotellerie und den örtlichen Einzelhandel. Indirekt profitieren weitere Branchen der Region durch Vorleistungen in Höhe von über 3 Mio. € von der Wallfahrt. Letztlich induziert die Wallfahrtsstätte damit insgesamt eine Wertschöpfung von rund 2,4 Mio. €, die den regionalen Betrieben sowohl als Gewinn als auch für Löhne und Gehälter zur Verfügung stehen. Im zweiten Fall entspräche dies einem Beschäftigungsäquivalent von fast 80 Arbeitsplätzen.

Maria Vesperbild bietet ein dichtes Veranstaltungsangebot - der jährliche Wallfahrtskalender zählt über 100 Seiten. Rund 1.000 Gläubige nehmen jeden Sonntag an den Hl. Messen teil (Düren 2011), dennoch ist der Ausflug (48\%) noch vor dem Gottesdienst (42\%) das häufigste Motiv der jährlich rund 450.000 Besucher. Der hohe Anteil an Dauerbesuchern², die geringen Entfernungen ihrer Anreise und der sehr geringe Anteil an Übernachtungsgästen reduzieren zwar die individuellen Ausgaben der Besucher, die relativ große Besucherzahl führt dennoch zu einer Gesamtsumme von fast 13 Mio. $€$, die in die lokale Ökonomie, in erster Linie in die Gastronomie, fließen (Fischer 2019). Abzüglich Steuern und Vorleistungen induziert die Wallfahrtsstätte so eine Wertschöpfung von rund 6,2 Mio. €, die die Gastronomie- und Einzelhandelsbetriebe rund um die Wallfahrtskirche als Gewinne verbuchen oder in Löhne und Gehälter investieren können, was rechnerisch 220 Arbeitsplätzen entsprechen würde.

\section{Synopse}

Durchschnittlich geben Tagesgäste in Deutschland $29 €$ aus (Harrer \& Scherr 2013). Diesen Wert erreicht keine der untersuchten Destinationen (vgl. Tab. 1). Allenfalls Maria Brünnlein und Maria Vesperbild nähern sich mit 28 bzw. $27 €$ dieser Marke. Auch die durchschnittlichen Ausgaben der Übernachtungsgäste in Deutschland von $116 €$ können an keinem der Wallfahrtsorte erzielt werden. Offensichtlich geben Besucher von kleinen und mittleren Wallfahrtsstätten

2 Als Dauerbesucher werden hier Personen bezeichnet, die mehrmals pro Jahr die Wallfahrtsstätte aufsuchen. 
weniger Geld aus als Besucher von profanen Destinationen. Dies würde auch die etwas höheren Ausgaben in Maria Brünnlein und in Maria Vesperbild mit ihren vergleichsweise hohen Anteilen profan motivierter Besucher erklären. Einfluss auf die Wertschöpfung vor Ort hat aber auch die Zahl und der Anteil der Übernachtungsgäste. Deutschlandweit geben diese pro Tag rund $4 \mathrm{Mal}$ so viel aus als Tagesgäste aus, bei den untersuchten Wallfahrtsorten zumindest 3,6 Mal so viel.

Tabelle 1: Synopse der Fallstudien-Befunde (gerundete Werte)

\begin{tabular}{|c|c|c|c|c|}
\hline & Biberbach & Wigratzbad & $\begin{array}{l}\text { Maria } \\
\text { Brünnlein }\end{array}$ & $\begin{array}{l}\text { Maria } \\
\text { Vesperbild }\end{array}$ \\
\hline Einwohnerzahl & 1.900 & 250 & 2.540 & 30 \\
\hline Besucherzahl (pro Jahr) & 10.000 & 15.000 & 150.000 & 450.000 \\
\hline $\begin{array}{l}\text { Ausgaben der Tagesgäste } \\
\text { (pro Tag und Kopf) }\end{array}$ & $3 €$ & $20 €$ & $28 €$ & $27 €$ \\
\hline $\begin{array}{l}\text { Ausgaben der Über- } \\
\text { nachtungsgäste } \\
\text { (pro Tag und Kopf) }\end{array}$ & - & $68 €$ & $105 €$ & $95 €$ \\
\hline $\begin{array}{l}\text { Anteil Übernachtungs- } \\
\text { gäste }\end{array}$ & $0 \%$ & $78 \%$ & $12 \%$ & $2 \%$ \\
\hline $\begin{array}{l}\text { Möglichkeiten für } \\
\text { Ausgaben }\end{array}$ & Gaststätte & $\begin{array}{l}\text { Gaststätten } \\
\text { Beherbergung } \\
\text { Devotionalien- } \\
\text { läden } \\
\text { Einzelhandel } \\
\text { Seminare/ } \\
\text { Exerzitien }\end{array}$ & $\begin{array}{l}\text { Gaststätte } \\
\text { Beherbergung } \\
\text { Devotionalien- } \\
\text { laden } \\
\text { Einzelhandel } \\
\text { Seminare/ } \\
\text { Exerzitien }\end{array}$ & $\begin{array}{l}\text { Gaststätten } \\
\text { Beherbergung } \\
\text { Devotionalien- } \\
\text { laden }\end{array}$ \\
\hline Bruttoumsatz (pro Jahr) & $29.000 €$ & $431.000 €$ & $5.539 .000 €$ & $12.870 .000 €$ \\
\hline $\begin{array}{l}\text { Einkommenseffekt } \\
\text { (pro Jahr) }\end{array}$ & $15.000 €$ & $187.000 €$ & $2.374 .000 €$ & $6.233 .000 €$ \\
\hline $\begin{array}{l}\text { Beschäftigungsäquivalent } \\
\text { (Arbeitsplätze) }\end{array}$ & 0,5 & 6,6 & 80 & 220 \\
\hline
\end{tabular}

Neben den nachfrageseitigen Faktoren ist aber auch das Konsumangebot vor Ort ausschlaggebend für die Wertschöpfung, wie das Beispiel Biberbach, wo trotz des hohen Anteils profan motivierter Besucher - aber mangels Konsumangebote nur wenig Kaufkraft gebunden wird. Indes sind die individuellen Ausgaben der Gäste in Maria Brünnlein am höchsten, denn die Wallfahrtskirche liegt nur fuß- 


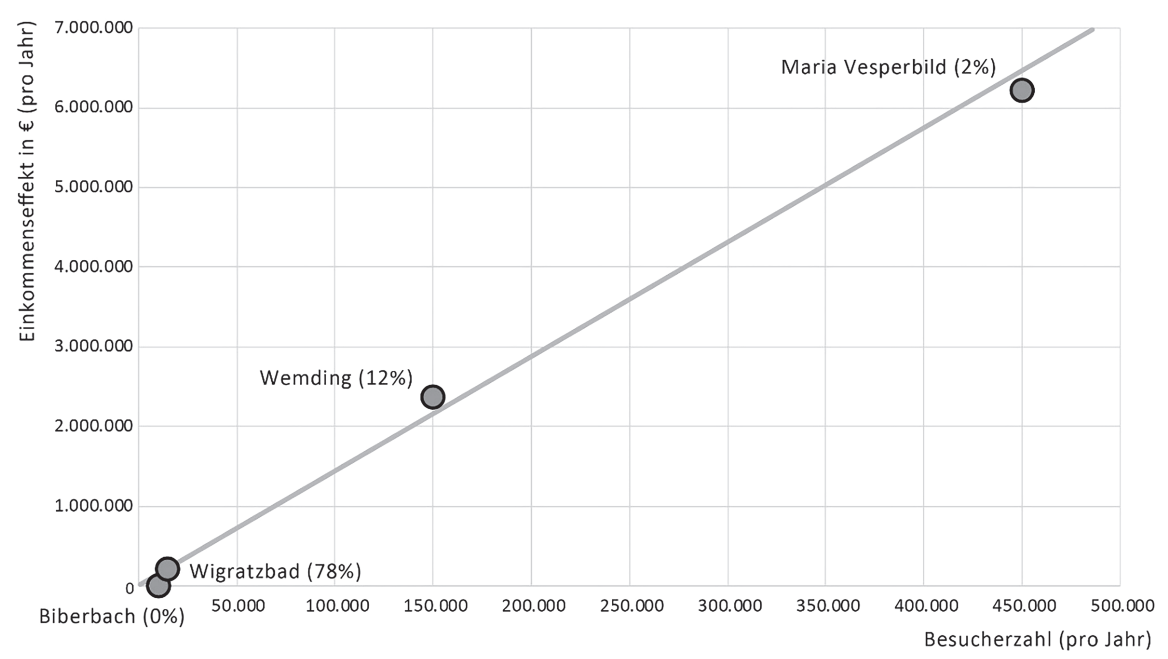

Abb. 1: Regionale Wertschöpfung und Besucherzahl an Wallfahrtsorten (sowie Anteil der Übernachtungsgäste)

läufig vom Zentrum der Stadt Wemding mit ihrem vielseitigen Konsumangebot entfernt. Mit den Ausgabemöglichkeiten und mit dem höheren Anteil an Übernachtungsgästen kann auch erklärt werden, warum in Wigratzbad fast $15 \mathrm{Mal}$ mehr Umsatz als in Biberbach erzielt wird, obwohl die Besucherzahl nur 1,5 Mal höher ist.

Trotz unterschiedlicher Ausprägungen der, die Wertschöpfung beeinflussenden Faktoren, zeigen aber die vier, in dieser Studie untersuchten Wallfahrtsorte einen fast linearen Zusammenhang zwischen Besucherzahl und Einkommenseffekt. Diese Proportionalität wird graphisch (vgl. Abb. 1) durch die geringen euklidischen Distanzen zur Regressionsgerade innerhalb der Punktewolke sichtbar. Der regionalökonomische Effekt scheint linear mit der Besucherzahl zuzunehmen. Nur en détail wirkt sich der etwas höhere Anteil der Übernachtungsgäste in Wemding (12\%) und Wigratzbad (78\%) positive auf das Einkommen aus. Wird ein linearer Zusammenhang angenommen, gilt für die Schätzung der regionalen Wertschöpfung (W) aus der Besucherzahl (B) die Formel $W=14 * B$ $+14.583,25$. Damit kann auf Basis der vier untersuchten Fallbeispiele allein aus der Frequentierung näherungsweise der Einkommenseffekt von Wallfahrtsorten mit einem Bestimmungsmaß von $\mathrm{R}^{2}=1$ geschätzt werden. ${ }^{3}$

30 = sehr ungenau; 1 = sehr genau 


\section{Fazit}

Wallfahrtsorte sind touristisch relevante Destinationen. In drei der vier untersuchten Fallbeispiele dominieren nicht-religiöse Besuchsmotive. Profane Touristen geben durchschnittlich mehr Geld als Wallfahrer aus, davon profitieren jene sakralen Destinationen, die auch für nicht- und wenig-gläubige Menschen von Interesse sind. Merklich steigen dabei die Ausgaben, wenn die Reise mit einer Übernachtung verbunden ist und wenn auch vor Ort entsprechende Konsumangebote vorhanden sind. Für die Höhe der lokalen Wertschöpfung durch die Besucher einer Wallfahrtsstätte sind somit letztlich vier Faktoren entscheidend: Die Besucherzahl, der Anteil profan motivierter Besucher, der Anteil der Übernachtungsgäste sowie die Konsumangebote vor Ort.

\section{Quellen}

Casanova, J. (2006): Religion, European secular identities, and European Integration. In Byrnes T.A., Katzenstein P.J. (Hrsg.), Religion in an expending Europe (S. 65-92). Cambridge: Cambridge University Press

Davie, G. (2008): From believing without belonging to vicarious religion: Understanding the patterns of religion in modern Europe. In Pollack, D. \& Olson, D. (Ed.), The role of religion in modern societies (S. 165-176). New York: Routledge

Düren P. C. (2011): Maria Vesperbild - Ein Wallfahrtsort mit einer gesunden Volksfrömmigkeit. In Buhlmann N.U., Styra P. (Hrsg.): Signum in Bonum (S. 869-881). Regensburg: Pustet Friedrich.

Edles, L. D. (2015): International Voluntourism as Secular Pilgrimage: A Case Study of Hosts and Guests in a Small Panamanian Town. International Journal of Religious Tourism and Pilgrimage, 3 (2), 57-64.

Fischer, M. (2019): Regionalökonomische Effekte von Wallfahrtsorten. Lokale Wertschöpfung am Beispiel maria Vesperbild. (Nicht veröffentlichte Master-Arbeit). Universität Augsburg.

Graave, E.J.E., Klijs, J., Heijman, W. (2017): The Economic Impact of Pilgrimage: An economic impact analysis of pilgrimage expenditures in Galicia. International Journal of Religious Tourism and Pilgrimage, 5 (3), 39-54.

Harrer B., Scherr S. (2013): Tagesreisen der Deutschen. Schriftenreihe des DWIF, 55.

Hilpert M. (2018): Regional Economic Impact of Small-Scale Pilgrimage Sites: Local Value Added by Small Sancturies in Rural Areas (Bavaria, Germany). International Journal of Religious Tourism and Pilgrimage, 6 (3), 81-85.

Imkamp W. zit. nach Renkikowski C (2010): Wallfahrt: Die älteste Form des Massentourismus. Abgerufen am 06. Juni 2010 http://www.katholisch.de/Nachricht.aspx?NId=1852

Laznik, E. (2019): Regionalökonomische Effekte von Wallfahrtsorten. Regionale Wertschöpfung am Beispiel Wemding. (Nicht veröffentlichte Bachelor-Arbeit). Universität Augsburg.

Marine-Roig E. (2015): Religious Tourism versus Secular Pilgrimage: The Basilica of La Sagrada Familia. International Journal of Religious Tourism and Pilgrimage, 3 (1), 25-37. 
Maschke J. (2005): Tagesreisen der Deutschen. Schriftenreihe des DIWF, 50.

Metzler D. (2007): Regionalwirtschaftliche Effekte von Freizeitgroßeinrichtungen. Eine methodische und inhaltliche Analyse. Kallmünz: Lassleben.

Pack, S. D. (2010): Revival of the Pilgrimage to Santiago de Compostela: The Politics of Religious, National, and European Patrimony, 1879-1988. The Journal of Modern History, $82,335-67$.

Rütter H., Guhl D.; Müller H. (1996): Wertschöpfer Tourismus. Ein Leitfaden zur Berechnung der touristischen Gesamtnachfrage, Wertschöpfung und Beschäftigung in 13 pragmatischen Schritten. Bern: Rüschlikon.

Sommer, A. (2012): Spiritueller Tourismus als eigenständige Tourismusart? Versuch einer Definition und Einordnung. Schriften zu Tourismus und Freizeit, 14, 21-46.

Wagner F. (2017): Regionalökonomische Effekte von Wallfahrtsorten. Regionale Wertschöpfung am Beispiel Wigratzbad. (Nicht veröffentlichte Bachelor-Arbeit). Universität Augsburg.

Wiltshier, P. \& Griffiths, M. (2016). Management practices for the development of religious tourism sacred sites. International Journal of Religious Tourism and Pilgrimage, 4 (7), 1-8.

Woodhead, L. J. P. (2016): Intensified religious pluralism and de-differentiation: the British example Woodhead. Society, 53 (1), 41-46.

\title{
Autoreninformationen
}

\author{
PD Dr. Markus Hilpert \\ Universität Augsburg \\ Institut für Geographie \\ Alter Postweg 118 \\ D - 86159 Augsburg \\ E-Mail: markus.hilpert@geo.uni-augsburg.de
}

Akademischer Oberrat und Leiter der Fachgruppe Standortentwicklung am Institut für Geographie der Universität Augsburg, markus.hilpert@geo.uni-augsburg.de Markus Hilpert studierte Geographie, Soziologie sowie Raumordnung, Landes- und Regionalplanung. Nach dem Studium arbeitete der Diplom-Geograph am Internationalen für Institut für Empirische Sozialökonomie (INIFES) als Projektgruppenleiter im Bereich der Politikberatung. Seit 2003 lehrt und forscht er an der Universität Augsburg. Seine Projekt- und Publikationsschwerpunkte liegen in den Bereiche Standortentwicklung, Tourismus, Kulturlandschaft und Religionsgeographie. 